\title{
Associação da fotobiomodulação e da hialuronidase tópica no extravasamento e infiltração de antineoplásicos. Estudo retrospectivo
}

RESUMO | Objetivo: avaliar a ação da fotobiomodulação associada a hialuronidase tópica nos extravasamentos e infiltrações de antineoplásicos, na prevenção de formação de lesões. Método: Estudo transversal, retrospectivo, descritivo que analisou os dados referentes a extravasamento e infiltração de antineoplásicos em um Ambulatório de Oncologia de um Hospital Público do interior do Estado de São Paulo no período de janeiro de 2018 a outubro de 2019. Resultados: A população do estudo constituise de 15 participantes, destes, 53\% $(n=8)$ apresentaram extravasamento e $47 \%(n=7)$ infiltração, gerando uma incidência de $0,6 \%$ e $0,5 \%$ respectivamente. Os antineoplásicos mais envolvidos foram paclitaxel e carboplatina. Não houve formação de lesões nos 15 pacientes estudados. Foram realizadas apenas 02 sessões de FBM em cada paciente com exceção de 01 paciente que foram realizadas 07 sessões, por ser extravasamento de antraciclina. Conclusão: O protocolo administrado foi efetivo na prevenção de formação de lesões durante extravasamento e infiltrações de antineoplásicos.

Palavras-chaves: Terapia com luz de baixa intensidade; Lasers; Extravasamento de materiais terapêuticos e diagnósticos; Antineoplásicos.

\begin{abstract}
Objective: to evaluate the action of photobiomodulation associated with topical hyaluronidase on extravasations and infiltrations of antineoplastic agents, in preventing the formation of lesions. Method: Cross-sectional, retrospective, descriptive study that analyzed data referring to extravasation and infiltration of antineoplastic agents in an Oncology Outpatient Clinic of a Public Hospital in the interior of the State of São Paulo from January 2018 to October 2019. Results: The population the study consists of 15 participants, of which $53 \%(n=8)$ had leakage and $47 \%(n=7)$ infiltration, generating an incidence of $0.6 \%$ and $0.5 \%$ respectively. The most involved antineoplastic agents were paclitaxel and carboplatin. There was no lesion formation in the 15 studied patients. Only 02 sessions of FBM were performed in each patient with the exception of 01 patient who were performed 07 sessions, for being anthracycline leakage. Conclusion: The administered protocol was effective in preventing the formation of lesions during extravasation and infiltration of antineoplastic agents.
\end{abstract}

Keywords: Low-Level Light Therapy, Lasers; Extravasation of Diagnostic and Therapeutic Materials; Antineoplastic Agents.

RESUMEN I Objetivo: evaluar la acción de la fotobiomodulación asociada a la hialuronidasa tópica sobre extravasaciones e infiltraciones de agentes antineoplásicos, en la prevención de la formación de lesiones. Método: Estudio descriptivo, transversal, retrospectivo, que analizó datos referentes a extravasación e infiltración de agentes antineoplásicos en un Ambulatorio de Oncología de un Hospital Público del interior del Estado de São Paulo de enero de 2018 a octubre de 2019. Resultados: La población el estudio consta de 15 participantes, de los cuales el 53\% $(n=8)$ presentaba fugas y el $47 \%(n=7)$ infiltración, generando una incidencia de 0,6\% y 0,5\% respectivamente. Los agentes antineoplásicos más implicados fueron paclitaxel y carboplatino. No hubo formación de lesiones en los 15 pacientes estudiados. Solo se realizaron 02 sesiones de FBM en cada paciente con excepción de 01 paciente que se realizaron 07 sesiones, por ser fuga de antraciclinas. Conclusión: El protocolo administrado fue eficaz para prevenir la formación de lesiones durante la extravasación e infiltración de agentes antineoplásicos. Palabras claves: Terapia por Luz de Baja Intensidad; Rayos Láser; Extravasación de Materiales Terapéuticos y Diagnósticos; Antineoplásicos.

\section{Karina Alexandra Batista da Silva Freitas}

Enfermeira, mestre e doutoranda pela Faculdade de Medicina de Botucatu UNESP. Supervisora do Ambulatório de Oncologia do Hospital Estadual Botucatu. Botucatu (SP).

ORCID: 0000-0002-3413-8468

\section{Talita Oliveira de Lima}

Enfermeira, mestre pela Faculdade de Medicina de Botucatu - UNESP. Enfermeira assistencial do Ambulatório de Oncologia do Hospital Estadual Botucatu. Botucatu (SP). ORCID: 0000-0002-6617-6514

Recebido em: 06/09/2020

Aprovado em: 23/09/2020

\section{Eliana Maria Minicucci}

Dentista, doutora em Bases Gerais da Cirurgia pela Faculdade de Medicina de Botucatu - UNESP. Dentista do Ambulatório de Oncologia do Hospital Estadual Botucatu. Botucatu (SP).

ORCID: 0000-0002-4664-6929

\section{Karen Aline Batista da Silva}

Enfermeira, doutora pela Faculdade de Medicina de Botucatu - UNESP. Diretora do Núcleo de Apoio à Superintendência Hospital das Clínicas da Faculdade de Medicina de Botucatu (SP).

ORCID: 0000-0002-8643-5333

\section{Alessandra Passarelli Vigliassi}

Enfermeira especialista em oncologia, membro do TIME de cateter do Hospital das Clínicas da Faculdade de Medicina de Botucatu - UNESP. Enfermeira assistencial do Ambulatório de Oncologia do Hospital Estadual Botucatu. Botucatu (SP). ORCID: 0000-0002-4651-888X

\section{Regina Célia Popim}

Professora associada do Departamento de Enfermagem da Faculdade de Medicina de Botucatu - UNESP.

ORCID: 0000-0001-8341-1590 
INTRODUÇÃO

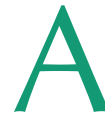
quimioterapia antineoplásica é amplamente utilizada no tratamento do câncer. Os quimioterápicos são classificados em vesicantes, irritantes e não vesicantes. O extravasamento refere-se ao escape do quimioterápico vesicante para fora do vaso, apresentando uma incidência de 0,1 a 7\% em infusão periférica, já a infiltração relaciona-se ao escape dos irritantes e não vesicantes. Os irritantes podem causar sintomas como ardor, flebite ou dor mesmo quando infundidas adequadamente e raramente causam necrose ou ulceração mesmo quando infiltradas em grandes quantidades $^{1,2}$. Por outro lado, as vesicantes quando extravasadas causam grandes danos ao tecido subjacente, provocando dor e levando rapidamente a necrose ${ }^{1,2,3}$. Diferentemente das drogas citadas anteriormente, as não vesicantes não causam danos teciduais ${ }^{3,4}$. Nesse sentido, um importante aspecto a ser investigado é como evitar complicações do extravasamento e das infiltrações.

A hialuronidase é um antídoto utilizado no extravasamento de quimioterápicos, no entanto é especifico para os vesicantes não ligante ao DNA, embora seja um potencial antídoto para as antraciclinas. Modifica a permeabilidade do tecido pela hidrólise do ácido hialurônico e ajuda a dispersar os alcaloides da vinca do tecido promovendo a reabsorção ${ }^{5}$. No máximo em dez minutos após a aplicação, já é possível observar a ação da enzima, pelo aumento da difusão do líquido extravasado em uma área 3 a 5 vezes maior do que uma área não tratada. A permeabilidade do tecido é restaurada de 24 a 48 horas após a aplicação do antídoto ${ }^{6}$.

Outra terapia recomendada para acelerar o processo cicatricial e em diversas patologias, melhorando substancialmente a qualidade de vida do paciente, é a terapia por fotobiomodulação (FBM) ou Laser de Baixa Intensidade (LBI) $)^{7,8}$.

Possui ações anti-inflamatórias, analgésicas e de cicatrização, além de não causar efeitos colaterais ao organismo, como muitas drogas de escolha par diversos tipos de tratamento, caracterizando-se como um recurso terapêutico muitas vezes de primeira escolha ${ }^{9}$.

Os pacientes devem ser avaliados clinicamente para a aplicação da FBM e devem ser considerados alguns parâmetros para o sucesso da terapêutica, como comprimento de onda, fluência, densidade de potência, estrutura de pulso e tempo da luz aplicada. A escolha de qual parâmetro utilizar é muito importante e vai depender da especificidade de cada tratamento. Existe uma dose ideal de luz para qualquer aplicação em particular e doses maiores ou menores que esses valores ótimos podem não ter efeito terapêutico. Portanto, uma escolha errada desses parâmetros pode significar em eficácia reduzida do tratamento, ou mesmo em um resultado terapêutico negativo. A FBM é caracterizada por uma dose-resposta bifásica, onde doses mais baixas de luz são frequentemente mais benéficas que doses altas ${ }^{10}$.

Fato esse comprovado em estudo realizado para investigar os efeitos da FBM no reparo de lesões cutâneas em modelo animal utilizando-se o comprimento de onda de 658, 830 e 904 nm. Observou-se um aumento da celularidade, da neovascularização e nas fibras de colágeno tipo III no grupo que utilizou o comprimento de onda de $658 \mathrm{~nm}$. Os grupos com comprimento de onda maiores, apresentaram resultados semelhantes ao grupo controle que não havia recebido a FBM ${ }^{11}$.

Quando aplicado no tecido, o estímulo é absorvido pelas células que possuem fotorreceptores, podendo ser enzimas, moléculas de membrana celular ou qualquer outra estrutura que tenha afinidade pelo comprimento de ondas vermelho (660nm) e infravermelho $(880 \mathrm{~nm})^{8}$.

O estudo justifica-se pelo extravasamento/infiltração causar uma diminuição da qualidade de vida, podendo afetar a continuidade do tratamento antineoplásico decorrente de possíveis danos causados.

Tem como objetivo, avaliar a ação da FBM associada a hialuronidase tópica nos extravasamentos e infiltrações de antineoplásicos, na prevenção de formação de lesões.

\section{MÉTODO}

Estudo transversal, retrospectivo, des- critivo que analisou os dados referentes a extravasamento e infiltração de antineoplásicos em um Ambulatório de Oncologia de um Hospital Público do interior do Estado de São Paulo.

Foram considerados como critério de inclusão os pacientes que apresentaram extravasamento e infiltração de antineoplásicos no período de janeiro de 2018 a outubro de 2019, ficando excluídos os extravasamentos e infiltrações de medicamentos não quimioterápicos.

Os casos de extravasamento e infiltração de antineoplásicos foram registrados pelo enfermeiro diariamente em planilha da Instituição, anotados no prontuário eletrônico do paciente (PEP) e reportados ao Hospital Sentinela. A Instituição possui um protocolo de extravasamento e infiltração bem delimitado, onde todos os funcionários foram treinados. Utilizam como antídoto a hialuronidase creme (uso tópico) 65 UTR e a FBM.

A coleta e análise dos dados foram realizados no período de agosto a setembro de 2020 após aprovação pelo Comitê de Ética em Pesquisa, com o protocolo CAAE: 31726620.5.0000.5411.

Após preenchimento do Termo de Consentimento Livre e Esclarecido pelos pacientes com extravasamento e infiltração, foram analisados os dados provenientes das anotações de enfermagem inseridas no PEP e as fichas de Notificação de Eventos Adversos (FNEA).

Inicialmente, todos os pacientes foram identificados por meio da FNEA. Após, realizada uma avaliação do PEP para traçar o perfil dos pacientes (faixa etária, sexo, escolaridade, idade, diagnóstico), além do protocolo antineoplásico utilizado, sintomas apresentados e se todos foram tratados conforme o protocolo da Instituição.

Foi realizada a análise estatística descritiva e utilizadas medidas simples como: distribuição de frequências, percentuais e média. A discussão dos achados foi feita com base na literatura produzida sobre o tema. A incidência foi calculada dividindo o número casos de extravasamentos/infiltração pelo número quimioterapia realizada no período, multiplicados por 100 . 


\section{RESULTADOS}

A população do estudo constitui-se de 15 participantes, destes, 53\% ( $\mathrm{n}=8)$ apresentaram extravasamento e $47 \%(n=7)$ infiltração. Do total da amostra, 50\% eram indivíduos do sexo feminino. A faixa etária predominante foi 70 a 80 anos (33\%), com predominante foi o fundamental completo

Quanto ao diagnóstico, o câncer de mama foi o mais prevalente $(26,6 \%)$ seguido de pulmão, linfoma e esôfago com 13,3\% cada e reto, leucemia linfocítica aguda, endométrio, laringe e próstata com 6,6\%.

Os quimioterápicos que apresentaram média de 74,8 anos. O grau de escolaridade $40 \%(n=6)$. maior incidência de extravasamento e infiltração estão descritos na tabela 1 e 2 respectivamente. Os sinais e sintomas mais apresentados durante os eventos foram listados na tabela 3. Durante o período do estudo foram administradas 12.866 quimioterapias, gerando uma incidência de 0,6\% para extravasamento e $0,5 \%$ para infiltração.

Todos os prontuários constavam atendimentos prestados durante a constatação do extravasamento/infiltração, bem como o uso de compressas mornas ou frias. Em relação as compressas, todas foram realizadas corretamente conforme o tipo de quimioterápico envolvido.

Constatou-se que a conduta realizada pelo enfermeiro frente ao extravasamento foi corretamente descrita na ficha de notificação e no PEP de todos os pacientes. Esses cuidados estavam descritos como: parada imediata da infusão, manutenção do dispositivo, aspiração da droga residual, realização de FBM com técnica pontual utilizando-se 1 joule (J) vermelho com distância de $1 \mathrm{~cm}$ a cada ponto de aplicação, compressas quentes ou frias, elevação do membro, aplicação do antídoto hialuronidase. Os pacientes foram orientados quanto ao uso de hialuronidase 3 vezes ao dia, compressas geladas ou mornas e retorno no ambulatório para realização de FBM 3 vezes na semana.

Todos os pacientes (15) voltaram ao ambulatório para nova avaliação dois dias após o evento e não apresentavam os sintomas descritos anteriormente, recebendo alta após 2 sessões de FBM. Apenas um paciente foi acompanhado por 30 dias por tratar-se de extravasamento de antraciclinas (vesicante ligante ao DNA) recebendo FBM 3 x semana nas primeiras duas semanas (totalizando 7 sessões), sem formação de lesões.

\section{DISCUSSÃO}

Tabela 2. Descrição dos quimioterápicos envolvidos na infiltração. Botucatu -SP, 2020

\begin{tabular}{lccc} 
& Quimioterápico & N & $\%$ \\
\hline Carboplatina & 02 & 28,5 \\
\hline Gencitabina & 01 & 14,3 \\
\hline Rituximabe & 01 & 14,3 \\
\hline Oxaliplatina & 01 & 14,3 \\
\hline Ifosfamida & 01 & 14,3 \\
\hline Cisplatina & 01 & 14,3 \\
\hline Total & 07 & 100
\end{tabular}

Fonte: Banco de dados organizado pela pesquisadora

Tabela 3. Sinais e sintomas apresentados durante o extravasamento. Botucatu-SP. 2020

\begin{tabular}{|c|c|c|}
\hline Sinais e Sintomas & $\mathbf{N}$ & $\%$ \\
\hline Parada da infusão & 15 & 100 \\
\hline Ausência de retorno venoso & 15 & 100 \\
\hline Edema & 15 & 100 \\
\hline Hiperemia & 15 & 100 \\
\hline Dor & 15 & 100 \\
\hline Calor & 01 & 6,6 \\
\hline
\end{tabular}

Fonte: Banco de dados organizado pela pesquisadora
O Instituto Nacional do Câncer (INCA) estima 66 mil novos casos de câncer de mama feminina e 30 mil casos de câncer de pulmão por ano para o triênio 2020-2022. Com exceção do câncer de pele não melanoma, o câncer de mama é o mais incidente nas mulheres ${ }^{12}$.

A incidência de extravasamento reportada nas literaturas podem variar desde 0,01\% a $7 \%{ }^{13}$, corroborando com nossos achados e divergindo de estudo realizado também em Hospital Universitário que observou um índice de $23,6 \%$ de extravasamento ${ }^{2}$. O resultado de um extravasamento pode levar a complicações a longo prazo, incapacidade permanente do membro, mudança de prognóstico do paciente e diminuição da qualidade de vida ${ }^{13}$.

Os principais sinais e sintomas relacionados ao extravasamento são: diminuição do fluxo de soro ou parada total da infusão, ausência de retorno venoso, queimação, edema, vermelhidão, a dor pode ou não estar presente ${ }^{14}$. 
Observamos que todos os pacientes estavam com as anotações e documentações corretas quanto ao extravasamento e infiltração. Neste sentido, o COFEN por meio da Resolução 429/2012, assegura como responsabilidade dos profissionais de enfermagem, registrar em documentos apropriados as informações pertinentes a todo cuidado prestado ao paciente $^{15}$. Entretanto, a literatura aponta uma ausência de registro dos cuidados em 15,9\% das fichas e notificação de extravasamento ${ }^{16}$.

Por ser considerada uma emergência oncológica, o extravasamento necessita de intervenções rápidas e assertivas16. Para isso, o protocolo de extravasamento deve ser estruturado e baseado em evidências científicas, garantindo um atendimento ágil e de qualidade ${ }^{17}$.

Portanto, constatou-se que o protocolo utilizado no serviço estava de acordo com o sugerido pela literatura internacional. A Infusion Nursing Society recomenda que incialmente deve-se parar a infusão, não desconectar o dispositivo, aspirar o máximo da droga possível, realizar compressas frias ou mornas e aplicação do antídoto padronizado ${ }^{18}$.

A Instituição dispõe de um aparelho de FBM, incorporando essa prática em seu protocolo de extravasamento. Não há relatos na literatura sobre a FBM no extravasamento de antineoplásicos, porém, já é muito utilizada na prevenção e no tratamento de lesões. Um exemplo dessa terapêutica é o uso na mucosite oral induzida por tratamento oncológico (radioterapia e/ou quimioterapia), onde ocorre a promoção da analgesia e da cicatrização das lesões orais. Para obter uma resposta adequada o FBM deve ser realizado ao menos 3 vezes na semana ${ }^{19}$.

Em relação ao antídoto hialuronidase, em scoping review realizado por Melo, et al (2017)20, constatou-se que diversos artigos trazem que a enzima degrada o ácido hialurônico, melhorando a absorção de drogas extravasadas, podendo ser eficaz na prevenção da necrose da pele por extravasamento com alcaloides da vinca e em taxanos.
Destaca-se ainda que o dexrazoxane é o único antídoto cuja eficácia foi testada em ensaios clínicos (por meio de biópsia) para o tratamento de extravasamento de antraciclinas e o único aprovado pelo FDA e pela Comissão Europeia para esse tipo de tratamento ${ }^{21}$, porém no Brasil não existe antídoto padronizado. Apresenta uma eficácia de 98\% no tratamento do extravasamento por antraciclinas, porém, o uso deste antídoto ainda é inviável para o setor público, devido ao seu alto custo ${ }^{21}$.

\section{CONCLUSÃO}

Levando-se em consideração a gravidade dos extravasamentos e infiltrações ocorridos e a não formação de lesões, infere-se que a associação da hialuronidase com o laser de baixa intensidade, é eficaz no tratamento desses eventos, podendo ser considerado uma terapia adjuvante aliado as outras práticas já descritas.

\section{Referências}

1. Gonzalez T. Chemotherapy extravasations: prevention, identification, management, and documentation. Clin J Oncol Nurs. 2013;17(1):61-6.

2. Gozzo TO, Panobianco MS, Clapis MJ, de Almeida AM. Dermatological toxicity in women with breast cancer undergoing chemotherapy treatment. Rev Lat Am Enfermagem. 2010;18(4):681-7.

3. Boulanger J, Ducharme A, Dufour A, Fortier S, Almarinc K. Gestão do extravasamento de agentes anti-neoplásicos. Support Care Cancer. 2015,23:1459-71.

4. Sauerland C, Engelking C, Wickham R, Corbi D. Vesicant extravasation part I: mechanisms, pathogenesis, and nursing care to reduce risk. Oncol Nurs Forum. 2006;33(6):1134-40

5. Kesic V, et al. Melatonin Ameliorates Doxorubicin-induced Skin Necrosis in Rats. Annals of Plastic Surgery. Volume 65, Number 2, August 2010

6. Doellman D, Hadaway L, Bowe-Geddes LA, Franklin M, LeDonne J, Papke-O'Donnell $L$, et al. Infiltration and extravasation: update on prevention and management. J Infus Nurs. 2009:32(4):203-11.

7. Neto CPS, Freire Júnior 0 . Um Presente de Apolo: lasers, história e aplicações. Rev. Bras. de Ensino de Física, vol 39 n 12017.

8. Gomes CF, SchapochnikA. 0 uso terapêutico do LASER de baixa intensidade (FBM) em algumas patologias e sua relação com a atuação na Fonoaudiologia. Distúrb Comum, São Paulo, 29 (3): 570-578, set, 2017.

9. Gomes CF, Schapochnik A. The therapeutic use of low intensity laser (LLLT) in some diseases and its relation to the performance in speech therapy. Distúrb. comun. 2017;29(3):570-8. DOI: 10.23925/2176-2724.2017v29i3p570-578.

10. Chung H, Dai T, Sharma SK, Huang YY, Carroll JD, Hamblin MR. The nuts and bolts of Low-level Laser (Light) therapy. Ann Biomed Eng. 2012:40(2):516-33. DOI:10.1007/s10439-011-0454-7.

11. Barbosa LS, Parisi JR, Viana LC, Carneiro MB, Novaes RD, Sousa L. The photobiomodulation $(658,830$ and $904 \mathrm{~nm})$ on wound healing in histomorphometric analysis. Fisioter. Mov. 2020;33:e003318. DOI: 10.1590/1980-5918.033.A018.

12. Instituto Nacional de Câncer José Alencar Gomes da Silva. Coordenação de Prevenção e Vigilância. Estimativa 2020: incidência de câncer no Brasil / Instituto Nacional de Câncer José Alencar Gomes da Silva. Coordenação de Prevenção e Vi- gilância. - Rio de Janeiro: INCA, 2019. [Acesso em 09/09/2020] https://uww.inca. gov.br/sites/ufu.sti.inca.local/files//media/document//estimativa-2020-incidencia-de-cancer-no-brasil.pdf

13. Jackson-Rose J, Del Monte J, Groman A, Dial LS, Atwell L, Graham J, et al. Chemotherapy extravasation: establishing a national benchmark for incidence among Cancer Centers. Clin J Oncol Nurs. 2017;21(4):438-45.

14. Correia JN, Albach LSP, Albach CA. Chemotherapeutic's extravasation: knowledge of the nursing team. Revista Ciência e Saúde [Internet], 2011 Jan/June [cited 2011 Dec 12];4(1):22-31. Available from: http://revistaseletronicas.pucrs.br/ojs/index.php/ faenfi/article/view/9151/6627

15. Conselho Federal de Enfermagem (BR), Resolução COFEN no 429/2012. Dispõe sobre o registro das ações profissionais no prontuário do paciente, e em outros documentos próprios da enfermagem, independente do meio de suporte - tradicional ou eletrônico [online]. Conselho Federa de Enfermagem; 2012 [citado 2020 ago 12]. Available in: http://www.cofen.gov.br/resoluo-cofen-n-4292012_9263.html.

16. Gozzo TA, Almeida TD, Cruz LAP. Notificação de extravasamento de agentes quimioterápicos em um Hospital Universitário. Cienc Cuid Saúde.17(2), Abr-Jun, 2018. 17. Melo JM, Oliveira PP, Rodrigues AB, Souza RS, Fonseca DF, Gontijo TF, et al. Construção e avaliação de bundle frente ao extravasamento de antineoplásicos: estudo metodológico. Acta Paul Enferm. 2020; eAPE20190075.

18. Journal of Infusion Nursing Society. The Official Publication of the Infuson Nurses Society. Supplement toJanuary/February 201639 (1S)

19. Florentino ACA, Macedo DR, Davd EF, Carvalho K, Guedes CCFV. Tratamento da mucosite oral com laser de baixa potência: revisão sistemática da literatura. Rev. Ciênc. Med., Campinas, 24 (2):85-92, maio/ago., 2015.

20. Melo JMA, Oliveira PP, Souza RS, Fonseca DF, Gontijo TF, Rodrigues AB. Prevention and conduct before the Extravasation of antineoplastic chemotherapy: scoping review. Rev Bras Enferm. 2020;73(4):e20190008. doi: http://dx.doi.org/10.1590/00347167-2019-0008

21. Roe H. Anthracycline extravasations: prevention and management. Br J Nurs. 2011;20(17 Suppl 16):S8-22 